of caregiver training, home equipment inspections, and home nursing. Most families defaulted to the home discharge plan, although several families were unable to complete all discharge requirements by the time their child was medically stable and no longer needed inpatient level of care. This often caused discharge delays.

Objectives Save 100 PCU bed days by June 30, 2018 through enhancing discharge planning.

Methods A multidisciplinary team created a Discharge Planning Booklet, which was reviewed with new families. The booklet included eligibility criteria to be discharged to home, an eightweek checklist with all home discharge requirements, a map of TCCs in nearby states, and an opportunity to select an appropriate discharge plan.

Results For patients with new tracheostomies, the average PCU length of stay decreased from 77.5 days to 62.2 days, saving 15.3 days per patients. This saved 414 PCU bed days for 27 patients. Several process metrics were also impacted, including decreased training time and decreased days of discharge delay due to incomplete caregiver requirements.

Conclusions The project resulted in a unit culture change in the approach to discharge planning for this patient population. In June 2018, the intervention expanded outside of the PCU to three other units, impacting an additional 20 patients each year.

\section{IHI ID 02 SUSTAINING AN END TO HOMELESSNESS OVER TIME}

Beth Sandor, Lindsey Giblin. Community Solutions, USA

10.1136/ihisciabs.2
Background Sustainably ending homelessness requires multistakeholder teams to constantly solve for population level outcomes over time. Previously, no measurement framework existed for knowing if a community had reached an end to homelessness, and more importantly, if those gains were continually maintained.

Objectives In 2015, we began to use a sustaining threshold to measure if the communities that had reached functional zero for veteran homelessness had sustained their progress over time by continually developing new solutions to the everchanging complex problem of homelessness.

Methods Built for Zero communities that have reached functional zero submit seven monthly data points related to the number of actively homeless veterans entering and exiting their systems. We compare this monthly data to the community's original functional zero threshold to measure sustainability over time.

Results To date, eight communities have ended and sustained a measurable end to veteran homelessness. All continue to experience fluctuation in the number of veterans experiencing homelessness, after they reach functional zero. Four communities have sustained consistently, especially those with higher thresholds relative to their system size and capacity. Communities that have sustained for three months or more are more likely to have sustained long term.

Conclusions Sustaining an end to homelessness requires communities to continually track and respond to the dynamics of homelessness across a geographically defined area, even after they have 'ended' homelessness. Given the impact of social determinants on health outcomes, understanding how to maintain an end to homelessness has implications for populationlevel outcomes across housing and health sectors.

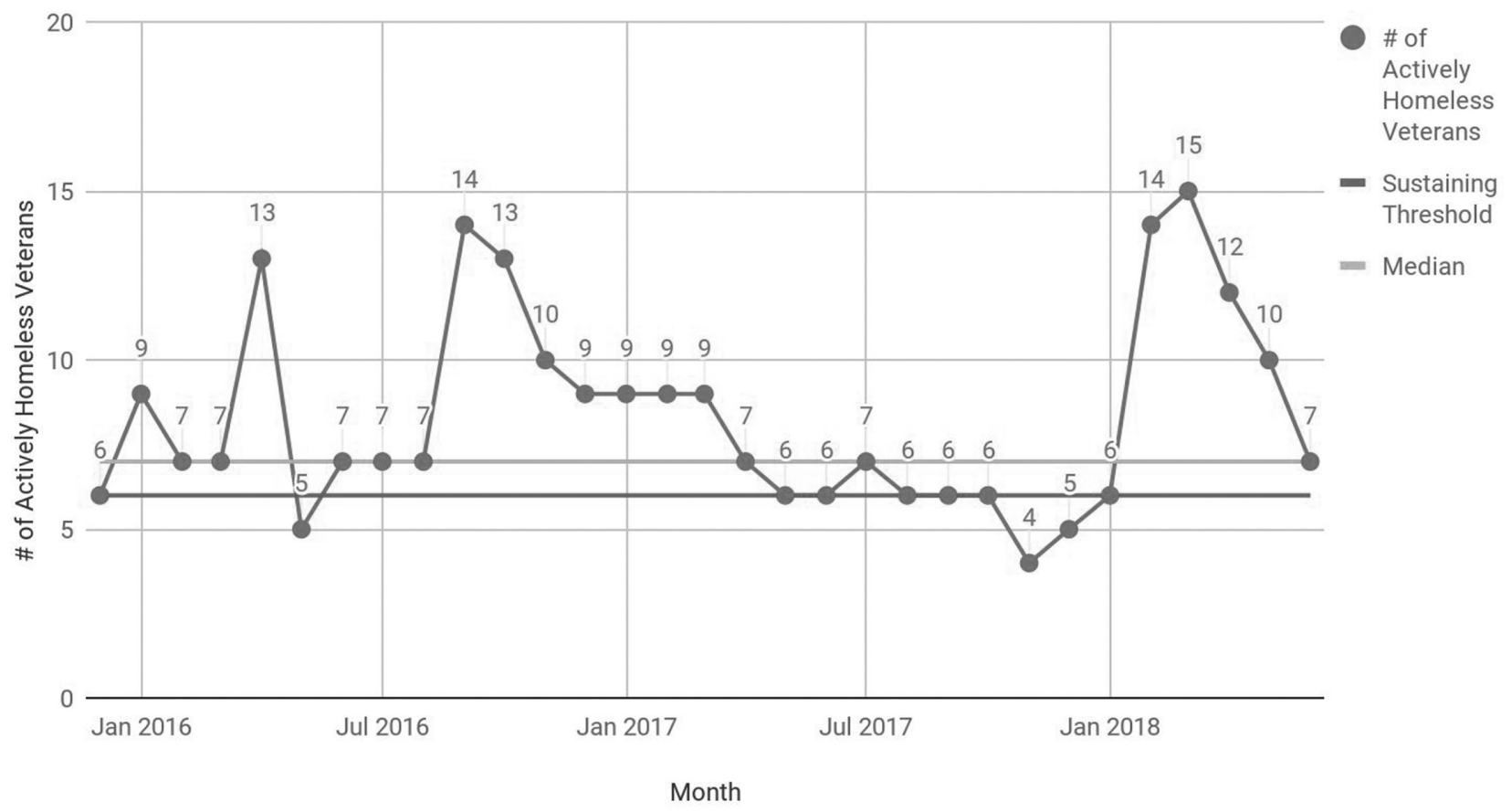

While not sustaining continually, the changes Montgomery County made to impact their system were effective according to run chart rules.

Abstract IHI ID 02 Figure 1 Veterans experiencing homelessness in montgomery country, MD 


\section{Abstracts}

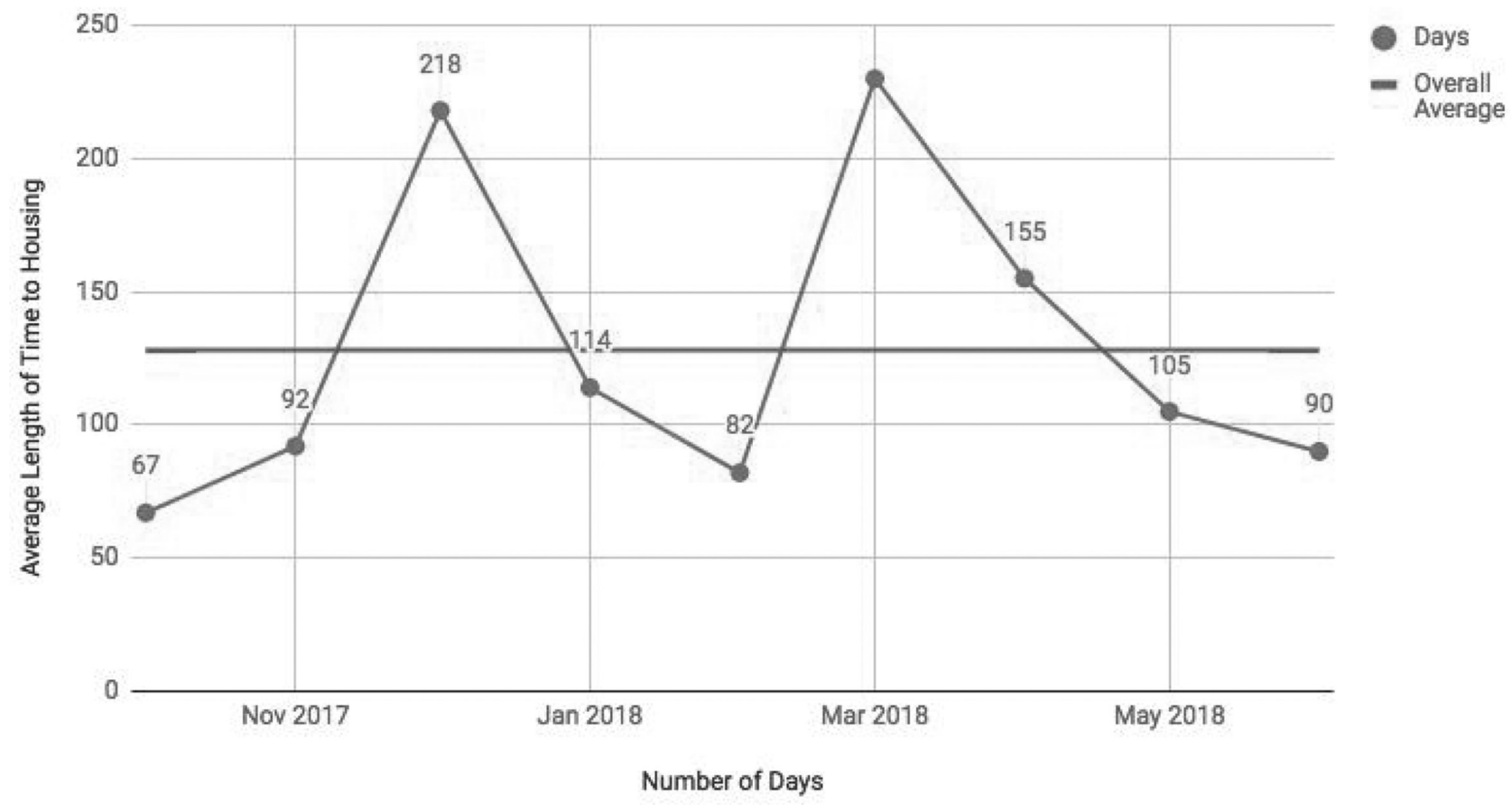

Abstract IHI ID 02 Figure 2 Average length of time from homeless identification to housing placement

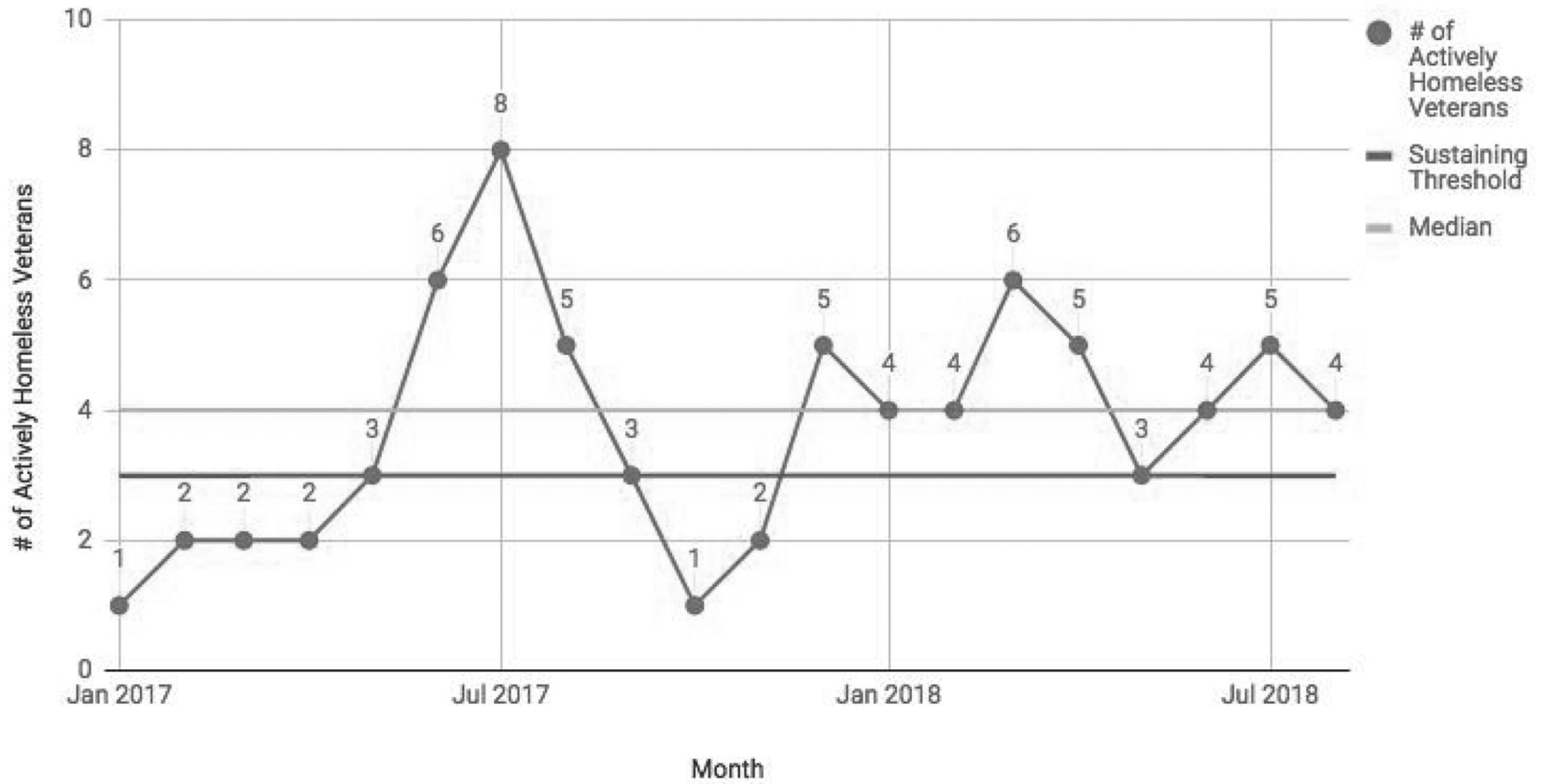

Norman has not sustained their functional zero threshold continually and according to run chart rules, the changes that Norman made to impact their system were not effective.

Abstract IHI ID 02 Figure 3 Veterans experiencing homelessness in norman, OK 


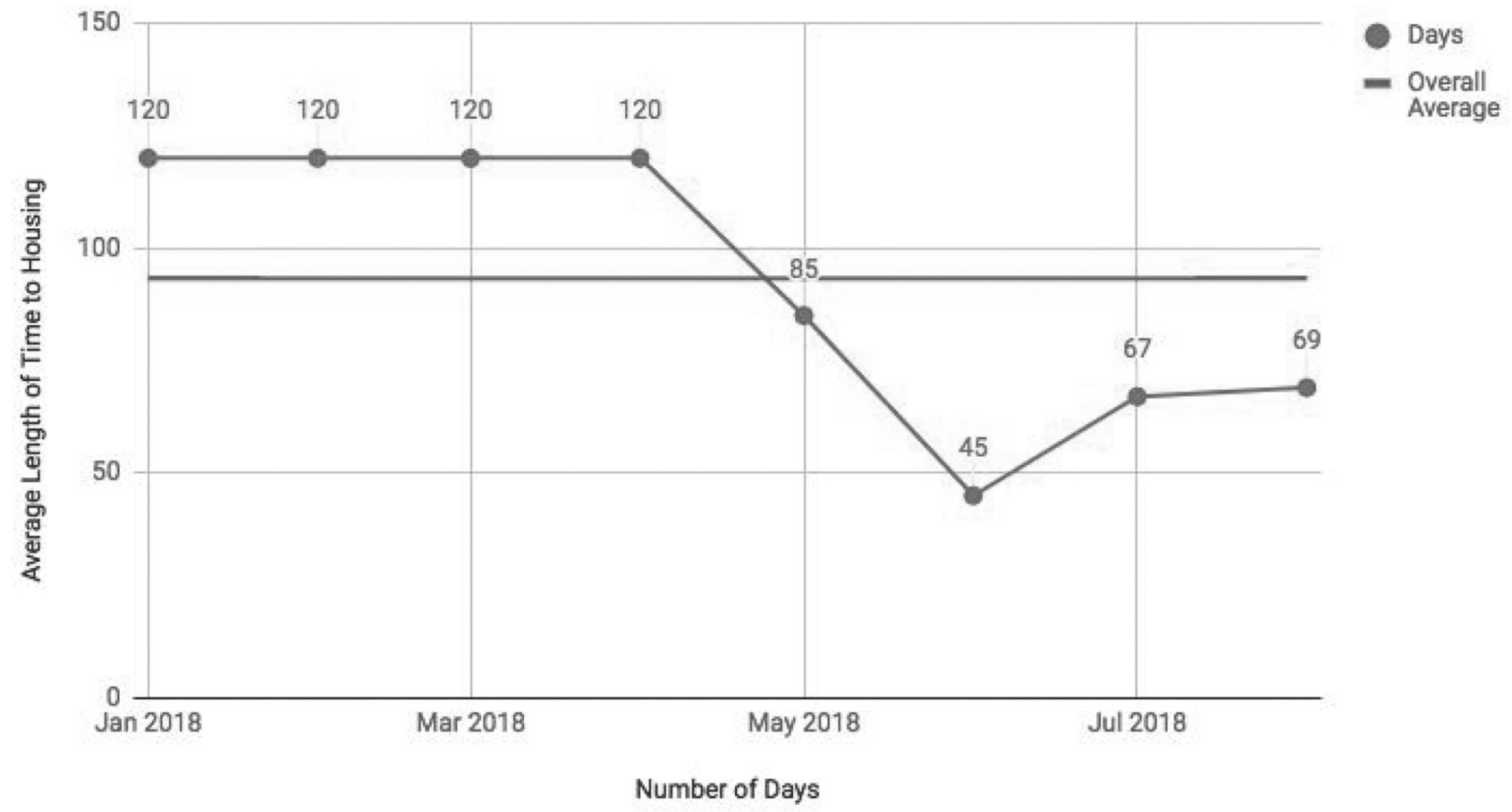

Abstract IHI ID 02 Figure 4 Average length of time from homeless identification to housing placement

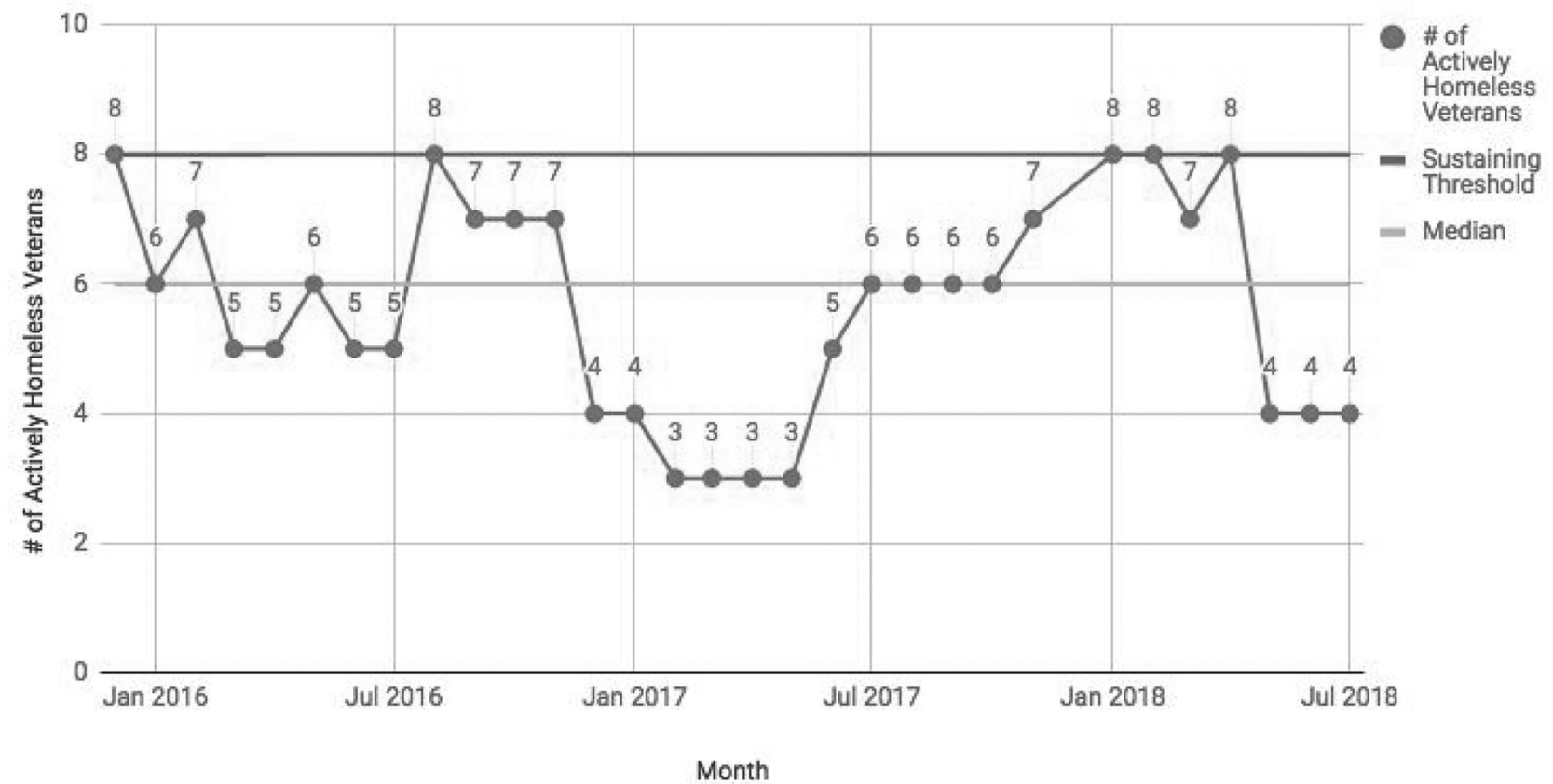

Rockford has sustained functional zero for two years, and the changes they made to impact their system were effective according to run chart rules.

Abstract IHI ID 02 Figure 5 Veterans experiencing homelessness in rockford, IL 


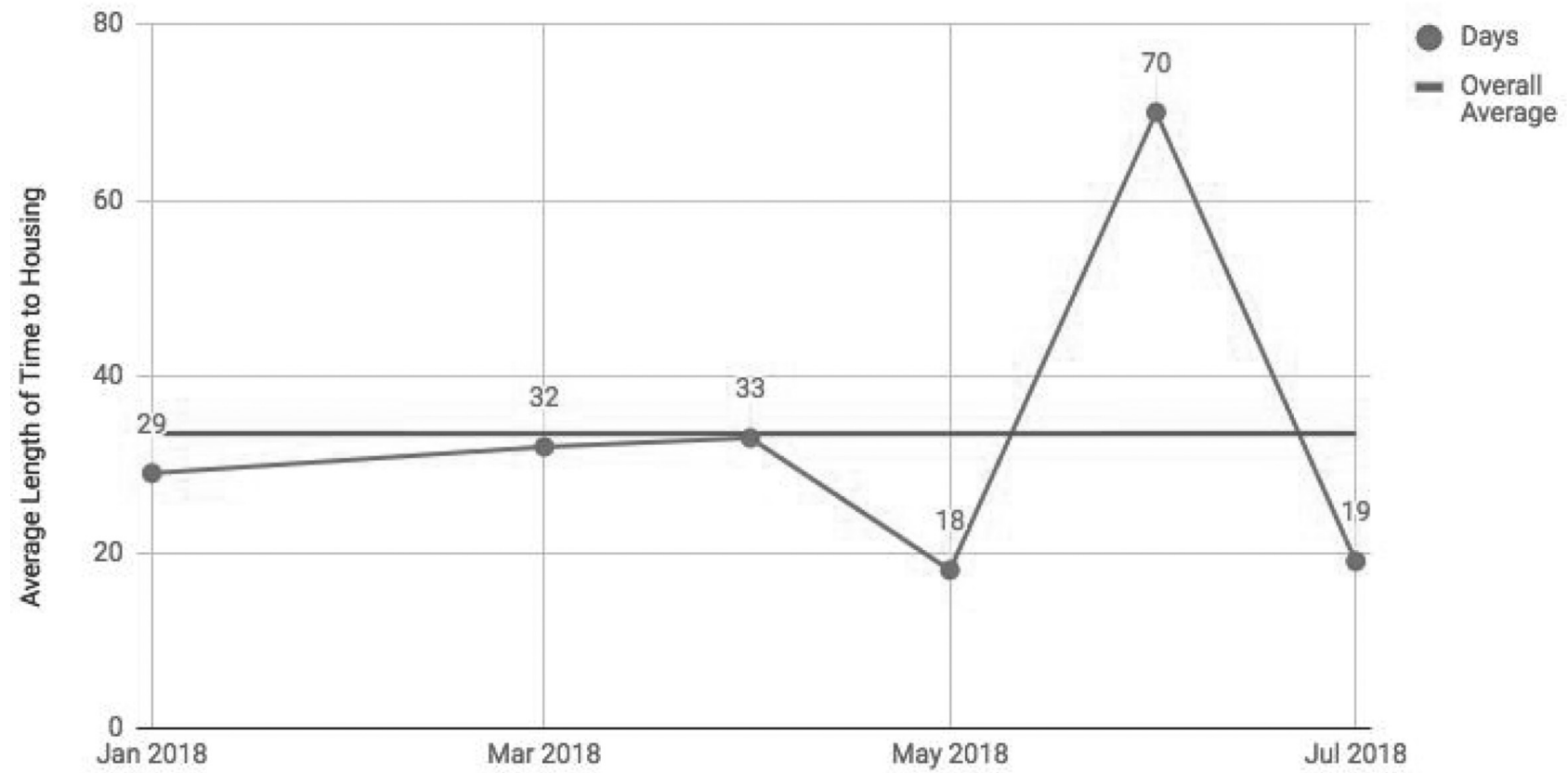

Number of Days

Abstract IHI ID 02 Figure 6 Average length of time from homeless identification to housing placement

\section{IHI ID 03 EQUIP, AN EVIDENCE-BASED QUALITY IMPROVEMENT PROCESS: IMPROVING THE SPEED TO INSIGHT}

Jose Benuzillo, Lucy Savitz. Kaiser Permanente, USA

\subsection{6/ihisciabs.3}

EQuIP, An Evidence-based Quality Improvement Process: Improving the Speed to Insight

\section{Literature monitoring in target clinical areas of importance for new comparative effectiveness evidence \\ - Comparative Effectiveness Studies \\ - Systematic Reviews \\ - Other Filtered Scientific Evidence}

Horizon scanning: structured approach that provides a systematic process for exploring dato

Provide parameters for guiding data exploration. Use prior available knowledge to define the scope and to add specificity to the data exploration.

Set the data exploration goals and build a logic model to describe the inputs and the outputs.
Assembling the needed data

from various sources to examine local practices

- EHR Data

- Clinical Databases

- Digital Data

- Claims Data

- Geospatial Data

- Patient-Reported Outcome Measures

Build a robust datoset by combining relevant data sources

Extract only the data relevant for the data exploration.

Spend time cleaning, merging, defining, and validating the data.

This should be a combined effort of

the data scientist and business or

clinical expert.

Try to fit the logic model with the data.
Background Despite the recognition that data can create value for healthcare organizations, only a few have adopted rigorous analytic approaches to support their data exploration efforts. With new sources and increasing amounts of data available to guide quality improvement work, having an evidence-based approach for directing data analytics is essential.
Data analytics

- Traditional Descriptive Statistics

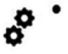

- Traditional QI Tools Root Cause Analysis Pareto Chart
SPC Charts Process Map

- Advanced Analytics

- Geospatial Analysis

Combine different tools to draw insight from your data

Identify patterns, anomalies, and opportunities. Seek to answer the following questions: Who?, When?, Where?, What?, How?, and Why?

Begin with broad descriptions and then focus on areas of value, such as unwarranted variation or worse than expected clinical outcomes. Risk

adjust and use benchmarks for meaningful comparisons.

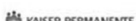
Center for Health Research 